\title{
Early Experiences with Indigenous Medicated Spacers in the Management of Infected Hip Prosthesis.
}

Pankaj Chand ${ }^{1}$, Sushil Rana Magar ${ }^{1}$, Bishnu Babu Thapa ${ }^{1}$, Bachhu Ram KC ${ }^{1}$, Amit Joshi ${ }^{1}$, Nirab Kayastha ${ }^{1}$.

${ }^{1}$ Department of Orthopedic Surgery, Shree Birendra Hospital.

\begin{abstract}
Introduction: Joint replacement surgery, has become one of the most frequent prosthetic surgeries over the past decades due to its success in restoring function to disabled arthritic individual. A two-stage revision arthroplasty is a well-accepted method for the treatment of a deep infection of a hip with a joint implant. In the present study, the results of three infected hips with the interim use of an indigenous cemented prosthesis along with gentamycin impregnated cement beads were assessed with a two stage revision.
\end{abstract}

Methods: Three consecutive patients who were managed with a two-stage revision hip arthroplasty for the treatment of a previous infection, with retained implants, were followed clinically and radiographically for an average of 15 months. Following removal of implants and debridement (first stage), an indigenous medicated prosthesis along with antibiotic coated cement beads were implanted, for an interim phase of six - eight weeks after which a new prosthesis was placed ( second stage ).In this period antibiotics ( both intravenous and oral) were administered to the patients.

Results: In a mean follow up period of 15 months, there is no evidence of recurrent infection in all three patients, as of now. The use of antibiotic coated cement prosthesis along with gentamycin mixed cement beads was associated with a satisfactory hip score, and better walking capacity in the interim period, a lower transfusion requirement at the time of reimplantation, and no postoperative dislocation.

Conclusions: Locally prepared medicated spacer and gentamycin coated beads help in eradicating hip infection, besides being more cost effective.

Keywords: indigenous, reimplantation, spacers.

\section{INTRODUCTION}

The current incidence of infection after primary total hip replacement is under one $\%$ in most large centers ${ }^{1}$ (Figure.1a) We report a short retrospective study of three previously infected hips treated with revision arthroplasty in two stages from July 2010 to December 2010, in our hospital and a private hospital outside.
Two-stage reimplantation remains the gold standard for the treatment of chronically infected hip today as the successful eradication of infection is well over $90 \% \%^{2,4,6,7,9}$. The use of temporary spacer prosthesis preserves softtissue tension and planes, making the reimplantation procedure much easier to perform ${ }^{2}$.In two-stage procedures, use of beads and either articulating or nonarticulating antibiotic-impregnated cement spacers also has lowered reinfection rates $^{3}$.

\section{Correspondence:}

Col. Dr. Pankaj Chand

Department of Orthopedics, Shree Birendra Hospital Kathmandu, Nepal.

Email: pankreena@hotmail.com

Phone: +977-9851092301 
We used indigenously prepared medicated hip prostheses. In the first stage we debrided the wound, removed all implants, and placed antibiotic mixed cement coated prosthesis along with antibiotic augmented cement beads(Figure. 1b,c). After 6-8 weeks, during which antibiotic therapy is given, the second stage is carried out, which consists of removal of beads and spacer, and implantation of a new prosthesis. We aim to assess the operated hips post operatively for function, and if there is any complication.

\section{METHODS}

Amongst the three infected hip patients, one of the patients was previously operated outside the country for a fracture dislocation of the acetabulum and underwent surgery abroad with a plate and screws. (Figure: 2a) The remaining two were treated at our hospital of which one was a total hip arthroplasty and the other was a unipolar hemiarthroplasty.

Firstly, patient demographics (age and sex and involved side ), date of any previous primary arthroplasty or any surgery regarding the hip, laboratory values of ESR, C-Reactive Protein (CRP), Complete Blood Count (CBC), electrolytes, blood sugar and radiographic information (x-rays, CT/MRI) were collected.

An ESR of greater than $40 \mathrm{~mm} / \mathrm{hr}$ and a CRP of greater than $10 \mathrm{mg} / \mathrm{l}$ were considered to indicate infection. ${ }^{1}$ All three were non-diabetic, afebrile, adult males. Pain was a common complaint and blood investigations were all consistent with infection, though external signs were not visible (such as an abscess or sinus/discharge or even a swelling).Even x-rays showed some loosening of the implants and changes in the joint area of the one with the acetabular fracture dislocation, all indicative of a chronic low grade infection. Pre-operative ESR and CRP were elevated in all three. All measures (such as antibiotics, rest, traction, and walking aids) were taken to treat the joints conservatively, but the joints did not respond satisfactorily. It was now that we decided to go for a two stage replacement arthroplasty. With this plan we thought of replacement spacers so as to treat the hips in the interim phase. Commercially available spacers (medicated once) are too expensive so we thought of the next best option. That is to prepare the medicated hips indigenously in the operation theatre only. We planned the cases pre operatively along with an anesthesiologist, cardiologist and a physician and took the patient's consent.

The making of an indigenous medicated spacer: a smaller sized (about two or three sizes smaller than the actual prosthesis ) Austin Moore/Thompson prosthesis was taken and covered with 20 gms of gentamycin mixed cement. The same cement was used to prepare the beads with a nylon suture acting as the thread.
In the first stage operation we performed a thorough debridement, and removal of all foreign bodies (prostheses/implants,). Samples were taken for gram stain, culture and HP analysis. Patients were kept on IV antibiotics for a week followed by another five weeks of oral antibiotics. Now the medicated hip spacer along with antibiotic augmented cement beads was placed. All operations were performed through a lateral approach (modified Hardinge).

In the second stage, medicated spacer along with gentamycin beads were removed after 6 to 8 weeks and revision hip arthroplasty done in all three cases. Three patients had final reimplantation, two were uncemented total hip replacements (J and J-American) )andone was a cemented bipolar hemi replacement arthroplasty (Green -Indian ). Of the uncemented hips, one was a revision femoral stem (Solution) with a $36 \mathrm{~mm}$ head in acetabular (Pinnacle) component while the other was a Corail femoral stem with a Duraloc acetabular component accommodating a $28 \mathrm{~mm}$ head.

Post- operatively, inj. Ceftriaxone (1gm BID) and inj. Cloxacillin (500 mg QID) were begun post operatively (one each of these were given at the time of operation too ). The intravenous antibiotics were given for a week all three cases, then shifted to oral drugs for a period of five weeks. Intravenous Enoxaparin ( $40 \mathrm{mg}$ ) subcutaneous was initiated after $12 \mathrm{hrs}$ and given every $24 \mathrm{hrs}$ for a period of five days (or once the patient became ambulatory). Getting up from side of the bed was begun from the second post operative day with gradual progression from partial weight bearing (with the aid of a walker/axillary crutches) to full weight bearing was as tolerated. Physiotherapy was begun from the first week with quadriceps drill and abductor drill from the third week.

\section{RESULTS}

CBC (total counts of red and white blood cells along with differential count of white blood cells; hemoglobin percentage) showed a normal picture in all throughout. Preoperatively ESR and CRP were elevated in all three (ESR $>45 \mathrm{~mm}$ and CRP $>25 \mathrm{mg} / \mathrm{L}$ ). Post-operatively they normalized six to eight weeks after re-implantation. Aspiration before surgery is a controversial issue, so we did not perform an aspiration before hand and made a decision based on clinical/radiological findings and CRP/ ESR values.

Intra operative Gram stain of all three showed growth of Staphylococcus aureus, and cultures were also positive in all three. Antibiotics were selected as per the culture and sensitivity in the three patients whose cultures came positive. Histopathological analysis showed chronic inflammatory cells, consistent with infection. 
At an average follow-up of 15 months (range 12 months to 20 months) no recurrence of infection or any complication occurred. As of writing this article the patients were without any pain and their range of motion was within functional limits. There were no complications (such as infection/dislocation) that needed an intervention. One patient with acetabular fracture dislocation had slight shortening of $1.5 \mathrm{~cm}$, because of higher acetabulum, that was from the time of primary surgery. They are asymptomatic till now. The younger patient in whom an uncemented THR was done, walks without any aid, while the other two walk with aid of a cane. All 3 of them are community ambulators and in fact one of them (young one with uncemented revision), is an active soldier in the army. We followed the patients for one year regularly. Thereafter only one patient (the army man) has come for regular follow ups. Mean Harris hip score was 30 (range 20 - 40) pre operatively, which improved to 75(range 70-85) till the last follow up. Of these one, the young soldier's function was good, while the other two were fair. We were certain that at the end of a longer follow-up they would have been even better. Like the young army man had a score of 85 when he last reported at the end of 18 months. Radiographs in all three showed no loosening at the end of the year and a well-fitting intact prosthesis (Table. 1).

Figure 1. X-Rays of cases
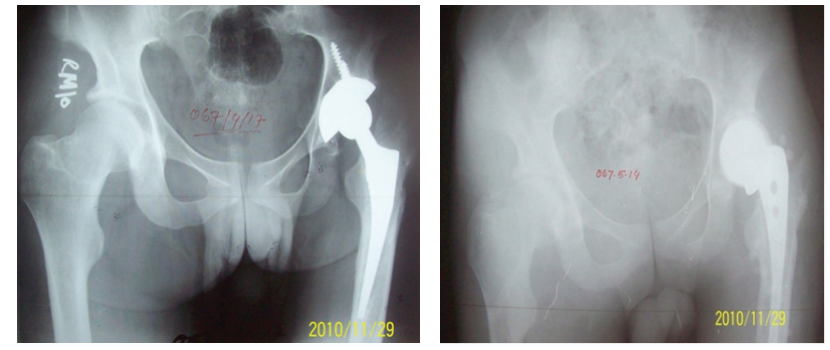

a. Uncemented THR done 2 years back

b. Previous implants removed and indigenous medicated spacer along with gentamycin beads were placed.

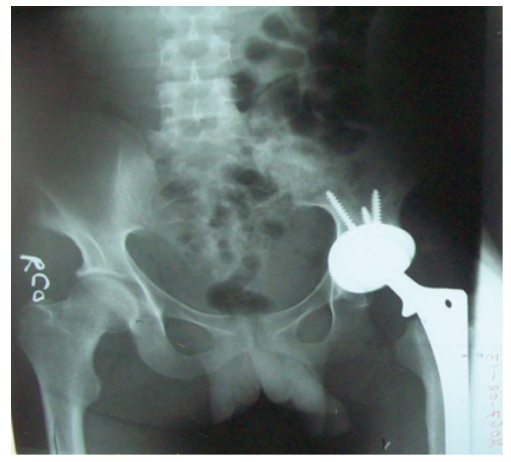

c. After 6 wks spacer along with the beads were removed and revision THR done

Table 1. Details of the patients

\begin{tabular}{|c|c|c|c|c|c|c|c|c|c|}
\hline 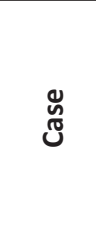 & 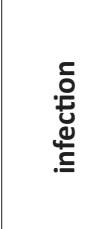 & $\begin{array}{l}n \\
0 \\
\mathbb{8} \\
0 \\
\frac{0}{+} \\
\frac{1}{d} \\
\frac{0}{0} \\
\frac{0}{n}\end{array}$ & 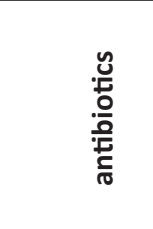 & 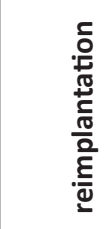 & 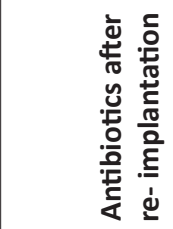 & 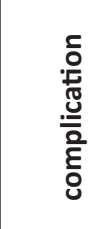 & $\begin{array}{l}\frac{\partial}{n} \\
\frac{\pi}{0} \\
\frac{0}{0} \\
\frac{2}{\frac{t}{2}} \\
\frac{t}{0}\end{array}$ & 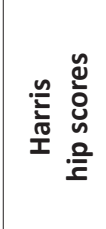 & $\begin{array}{l}\frac{0}{3} \\
3 \\
0 \\
0 \\
0\end{array}$ \\
\hline Case 1 & $19 \mathrm{~m}$ & Both & $\begin{array}{l}1 \text { wk iv } \\
5 \text { wks oral }\end{array}$ & 6 wks & $\begin{array}{l}3 \text { days iv } \\
7 \text { days oral }\end{array}$ & None & $\begin{array}{l}\text { Uncemented } \\
\text { THR }\end{array}$ & $\begin{array}{l}85 \\
\text { (good) }\end{array}$ & $20 \mathrm{~m}$ \\
\hline Case 2 & $9 \mathrm{~m}$ & Both & $\begin{array}{l}1 w k \text { iv } \\
5 \text { wks oral }\end{array}$ & $8 w k s$ & $\begin{array}{l}3 \text { days iv } \\
7 \text { days oral }\end{array}$ & None & $\begin{array}{l}\text { Uncemented } \\
\text { THR }\end{array}$ & $\begin{array}{l}70 \\
\text { (fair0 }\end{array}$ & $12 \mathrm{~m}$ \\
\hline Case 3 & $15 \mathrm{~m}$ & Both & $\begin{array}{l}1 \text { wk iv } \\
5 \text { wks oral }\end{array}$ & $8 w k s$ & $\begin{array}{l}3 \text { days iv } \\
7 \text { days oral }\end{array}$ & None & $\begin{array}{l}\text { Cemented } \\
\text { bipolar }\end{array}$ & $\begin{array}{l}72 \\
\text { (fair) }\end{array}$ & $14 \mathrm{~m}$ \\
\hline
\end{tabular}

\section{DISCUSSION}

In our study, two were right sided hip infections while one was a left sided. All were males with an average age of 48years (mean $25-65$ ). The two-stage reconstruction is an effective, safe technique even when the infection is caused by a virulent organism. ${ }^{5}$ In this study, mean time elapsed between the primary operation and the infection onset symptoms was fourteen months (range nine nineteen months).
Macdonald et al $^{5}$ did a two stage revision of an infected hip in 81 patients but with an interim period of resection arthroplasty. However, resection arthroplasty, even if used as a temporary method between stages, is associated with a considerable loss of function, and should be considered in patients unfit to have an additional reconstructive procedure and non cooperative patients. When talking about joint replacement, complications are a worry. The most frequent being aseptic loosening. But of greater concern is infection, the second most frequent 
complication. ${ }^{7}$ The declinein the level of C-reactive protein seems to be the best test for monitoring the effectiveness of antibiotic treatment. Whereas a two stage approach provides a high success rate (about 95\%) for eradication of the infection, it frequently results in major morbidity due to prolonged immobilization of the patient, who is typically elderly. ${ }^{7}$

Prosthetic joint infections are of four types. ${ }^{10}$ In our series, all three cases were diagnosed with a late chronic infection (type two), for which a two stage revision arthroplasty is indicated. In recent years, two-stage exchange arthroplasty has emerged as the gold standard for successful elimination of infection. With success rates averaging $82 \%$ to $96 \%$, this treatment method has both the highest and most consistent rate of infection eradication. ${ }^{9}$ Successful eradication of infection after single-stage exchange arthroplasty has been reported to average from $60 \%$ to $83 \%$ after total hip infections. ${ }^{9}$

Once the first stage was accomplished the average duration of the interim period, before the second stage, was $7.3 \mathrm{wks}$ (range $6-8 \mathrm{wks}$ ). Total duration of antibiotics in the interim phase was six weeks (one week intravenous and for 5 weeks oral). There is no clear consensus in the orthopedic forum as to the duration of antibiotic used intravenously during the interim phase, ranging from two weeks to six weeks . ${ }^{12}$ Total duration of antibiotics post re-implantation was ten days (three days IV and 7 days oral). It is suggested that patients be off antibiotics for a minimum of 2 weeks before obtaining intra-articular culture. ${ }^{8}$ It is recommended that multiple cultures be obtained at the time of reoperation in patients being assessed for periprosthetic joint infection. ${ }^{8}$

In both one and two-stage revision procedures for infection, antibiotic-impregnated cement clearly reduces the reinfection rate. Two-stage revision is more versatile for the strategy of infection eradication and for the choice of the type of reconstruction compared to one-stage revision. First, two-stage revision allows the assessment of response to the antibiotics selected. If the organism detected pre-operatively is different from the organism detected in the first stage, it is possible to change the antibiotic treatment. Second, the method allows reconstruction using either cementless or cemented component. ${ }^{12}$ Two-stage septic revision surgery is the most common method for treating infected endoprostheses.$^{12}$ The interval period between removing the infected prosthesis and reimplanting a new prosthesis is 6 days to more than 6yrs (in cemented revision) and from 6 wks to 37 wks (in cementless revision) duration . ${ }^{12}$ After primary joint operations, the rate of infection was
1.3 per cent of 23,519 hips and 2.0 per cent of 16,035 knees. After revision operations, the rate was 3.2 per cent of 7161 hips and 5.6 per cent of 2714 knees. ${ }^{13}$

There is mention of hip scores in literature (Harris/Oxford /dysplasia,to name a few), but we selected Harris hip score for our cases because of wide following and also for convenience.

\section{CONCLUSIONS}

The present study supports the safety and efficacy of the routine use of antibiotic-loaded cement prosthesis along with gentamycin impregnated cement beads in the interim between the stages of a two-stage revision procedure for the treatment of an infection at the site of a hip arthroplasty.

\section{REFERENCES}

1. Haddad FS, Muirhead-Allwood SK, Manktelow ARJ, Bacarese H. Two-stage uncemented revision hip arthroplasty for infection. J Bone Joint Surg. 2000;82-B:689-94.

2. Hsieh $\mathrm{PH}$, Shih $\mathrm{CH}$, Chang MC, Lee MS, Shih $\mathrm{HN}$, Yang WE. Two-Stage Revision Hip Arthroplasty for Infection : Comparison Between the Interim Use of Antibiotic-Loaded Cement Beads and Spacer Prosthesis. J Bone Joint Surg. 2004;86:1989-97.

3. Joseph TN, Chen AL, Di Cesare PE. Use of AntibioticImpregnated Cement in Total Joint Arthroplasty. J Am AcadOrthop Surg. 2003;11:38-47.

4. Chen WS, Ful TH, Wang JW. Two-stage Reimplantation of Infected Hip Arthroplasties. Chang Gung Med J. 2009;32:188-97.

5. McDonald DJ, Fitzgerald RH Jr, Ilstrup DM. Twostage reconstruction of a total hip arthroplasty because of infection. J Bone Joint Surgery Am. 1989;71 (6):828-34.

6. SukeikM, Haddad FS. Two-stage procedure in the treatment of late chronic hip infections - spacer implantation.Int J Med Sci.2009;6 (5):253-7.

7. Bernard L, Hoffmeyer $P$, Assal $M$, Vaudaux $P$, Schrenzel J, Lew D. Trends in the treatment of orthopaedic prosthetic infections. Journal of Antimicrobial Chemotherapy. 2004;53:127-9.

8. Valle CD, Parvizi J, Bauer TW, DiCesare PE, Evans RP, Segreti J et al. The Diagnosis of Periprosthetic 
Joint Infections of the Hip and Knee. J Bone Joint Surg Am. 2011;93:1355-7.

9. Hernigou P, Lachianette CHF, Jalil R, Batista SU, Guissou I, Poignard A. Treatment of Infected Hip Arthroplasty. The Open Orthopaedics Journal.2010;4:126-31.

10. Jacobs C, Christensen CP, Berend ME. Static and Mobile Antibiotic impregnatedCement Spacers forthe Management of ProstheticJoint Infection . J Am AcadOrthop Surg.2009;17:356-68.

11. Pignatti G, Nitta S, Rani N, Dallari D, Sabbioni G, Stagni C, Giunti A. Two Stage Hip Revision in
Periprosthetic Infection: Results of 41 Cases. The Open Orthopaedics Journal. 2010;4:193-200.

12. Fink B. Revision of late periprosthetic infections of total hip endoprostheses: pros and cons of different concepts. Int J Med Sci. 2009;6 (5):287-95.

13. Hanssen $A D$, Rand JA. Evaluation and Treatment of Infection at the Site of a Total Hip or Knee Arthroplasty. An Instructional Course Lecture, American Academy of Orthopaedic Surgeons.1998;80-A (6):910-22. 